\title{
Impact of a Magnesium Sulfate Loading Dose on Fetal Umbilical Artery Doppler Parameters in Women with Severe Preeclampsia
}

\author{
Muhamed H. Gad ${ }^{1 *}$ M.B.B.CH, Mofeed F. Muhamed ${ }^{2}$ MD, Muhamed A. Abdelmoaty ${ }^{2}$ MD.
}

\author{
* Corresponding Author: \\ Muhamed H. Gad \\ muhammedhe169@gmail.com \\ Received for publication April \\ 16, 2020; Accepted May 31, \\ 2021; Published online May 31, \\ 2021.
}

Copyright The Authors
published by Al-Azhar
University, Faculty of Medicine,
Cairo, Egypt. Users have the
right to read, download, copy,
distribute, print, search, or link
to the full texts of articles under
the following conditions:
Creative Commons Attribution-
Share Alike 4.0 International
Public License (CC BY-SA 4.0)..
doi: 10.21608/aimj.2021.69899.1450.
${ }^{1}$ Obstetrics and Gynecology
Department, Imbaba General
Hospital, Cairo, Egypt.
${ }^{2}$ Obstetrics and Gynecology
Department, Faculty of Medicine,
Al-Azhar University, Cairo, Egypt.

\begin{abstract}
Background: Preeclampsia is the most significant reason of maternal mortality throughout the world, as well as golden way to perinatal mortality and morbidity, affecting $2-8 \%$ of pregnancies.

Aim of the study: : To see how magnesium sulfate affects fetal umbilical artery Doppler changesin women suffering from extreme preeclampsia.

Patients and Methods: It is interventional (experimental) nonrandomized study was carried out in the department of gynecology of the al-hussein university hospital according to sample size. 50 women in this study were drawn from the emergency department and the Obstetrics outpatient clinic. Between April 2020 and January 2021, they were all diagnosed with extreme preeclampsia.

Results: The treatment of magnesium sulfate to pregnant women having serious preeclampsia and eclampsia minimize umbilical artery Doppler velocimetry indices by a statistically significant amount. In terms of different Doppler parameters, there was a considerable difference in the study group's umbilical artery Doppler parameters before and after magnesium sulfate treatment (mean values of Resistance Index, Pulsatility Index, and Systolic/Diastolic ratio in the study group).

Conclusion: to diagnose complications and evaluate fetal well-being in pregnant women suffering preeclampsia, clinical examinations should be backed up and verified by laboratory tests and ultrasonography. Doppler flow tests should be included in the sonographic review since they can help predict preeclampsia and determine fetal condition.
\end{abstract}

Keywords: Fetal Umbilical Artery; Magnesium Sulfate; Sever Preeclampsia.

Disclosure: The authors have no financial interest to declare in relation to the content of this article. The Article Processing Charge was paid for by the authors.

Research Support: RCT approval numbers: NCT04656067 and PACTR202101794541918

Authorship: All authors have a substantial contribution to the article.

\section{INTRODUCTION}

Preeclampsia is a pregnancy condition that affects many body systems. Also it is considered a leading reasons of maternal and fetal morbidity globally.The major clinical signs of the disorder are hypertension and proteinuria, which occurs after the 20 weeks of pregnancy in women who have never been hypertensive before ${ }^{1 .}$

Preeclampsia is more frequent in some parts of the world than in others. It is estimated that 1-2 \% in some European countries are affected; however, in some South American and African countries, the frequency can range from $10 \%$ to $15 \%{ }^{2}$.

If one or more of the following occur when the patient is confined to his or her bed: higher systolic blood pressure of $160 \mathrm{mmHg}$ or $110 \mathrm{mmHg}$ diastolic or high on two occasions at least 4 hours apart, preeclampsia is considered serious. In the absence of other renal disease, renal insufficiency is described as a serum creatinine concentration of more than 1.1

$\mathrm{mg} / \mathrm{dl}$ or a doubling of serum creatinine concentration. The disturbance of the brain or vision pulmonary edema, also known as cyanosis, is a condition that affects the lungs.Epigastric or right upper quadrant pain, abnormal liver function,thrombocytopenia and a new-onset headache that isn't sensitive to medication and can't be understood by any other diagnosis ${ }^{3}$.

The trophoblast invades the spiral artery in the placental bed during normal pregnancy, fuses with the vessel wall, and replaces the endothelium, muscular layer, and neural tissue. The spiral arteries are transformed from small muscular vessels to broad non-muscular channels as a result of these physiological changes, which are independent of maternal vasomotor regulation. The trophoblastic 
invasion of the affected maternal spiral arteries is considered to be the cause of preeclampsia. ${ }^{4}$.

When utero-placental blood flow decrease, preeclampsia symptoms appear in the fetal placental unit. Intrauterine growth restriction (IUGR), oligohydraminos, placental abruption, and nonreassuring fetal status observed on ante-partum surveillance by Doppler ultrasound are examples of such manifestations ${ }^{3}$.

Doppler tests of the uterine artery indicate increased (PI) in the first and second trimester of pregnancy, which may confirm abnormal placental perfusion in pregnancies doomed to preeclampsia ${ }^{4}$.

In gynecology, magnesium sulfate is widely used and is the preferred treatment for two major pregnancy complications: preeclampsia and pre-termlabor. Preeclampsia patients are given magnesium sulfate to prevent seizures ${ }^{5}$.

Doppler sonography has long been used to study fetal circulation because it can disclose vital information about the fetal well-being and neonatal prognosis in high-risk pregnant women.Doppler sonography is used to assess the effects of different medications using Doppler blood waveforms ${ }^{6}$.

Magnesium sulfate's homodynamic effects are extremely diverse.In women with extreme preeclampsia, studies show a substantial drop in blood pressure and Doppler parameters of the uterine, umbilical, and fetal middle cerebral arteries., further research is needed to determine the hemodynamic effects of this procedure on the uterine, umbilical, and fetal middle cerebral arteries using Doppler flow velocity in women suffering from serious preeclampsia. This data will assist to solidify the use of magnesium sulfate and define a more effective fetal monitoring protocol in these expecting mothers ${ }^{7}$.

\section{PATIENTS AND METHODS}

It was interventional (experimental) non randomized study was conducted at the gynecology department of Al-Hussein University Hospital according to sample size.In this study 50 women who participate in, came from the emergency department and the Obstetrics outpatient clinic. Extreme preeclampsia was found in all of them. The study took place between April 2020 and January 2021. The following criteria were used to select patients for the study: Extreme preeclampsia is diagnosed when one or more of the following conditions are met: gestational age; 28-36 weeks, gestational age; 28-36 weeks, gestational age; 28-36 weeks, gestational age; 28-36 weeks, gestational age ;28-36 weeks, gestational age; 28-36 weeks, gestational age Presence of renal insufficiency (when serum creatinine concentration more than $1.1 \mathrm{mg} / \mathrm{dl}$ or a doubling serum creatinine concentration in the absence of any renal disease, cerebral or visual disorder, pulmonary edema or cyanosis, epigastric or right upper quadrant pain, impaired liver function, and thrombosis

The following criteria were used to exclude patients from the study:Patients taking antiplatelet medications, such as a reduced dose aspirin,patients on anticoagulant therapy, such as heparin, patients with gestational age less than 28 weeks, multiple gestations, fetal abnormalities, any chronic diseases in the mother, particularly diabetes, renal disease, epilepsy, or CNS lesion. Both participants were counseled and given the opportunity to sign a written informed consent form.Total blood , RH, fasting blood glucose levels, 2-hour postprandial blood glucose, liver functions, kidney functions, and urine analysis were all routine baseline investigations. = Doppler experiments: Before and 20 minutes after intravenous administration of 6 grams of magnesium sulfate, the resistance index ,pulsatility index , and systolic/diastolic ratio of the umbilical artery were measured (loading dose). Magnesium Sulfate Regimen: The magnesium sulfate was administered as a loading dose of 6 grams intravenous more than 20 minutes, followed by an intravenous infusion at a rate of 1 gram per hour for 24 hours, with clinical tests every four hours.

The apparatus was used to conduct Doppler experiments (Logiq p3 with Doppler unit and convex linear transducer 3.5 MHZ). The study hypotheses that6 $\mathrm{g}$ magnesium sulfate injected intravenously for 20 minutes would improve umbilical artery efficacy. Doppler indices are a type of index that measures how fast something moves. The null hypothesis was that using a loading dose of magnesium sulfate had no statistically meaningful effect on umbilical artery Doppler.

\section{RESULTS}

Fifty patients were selected for our study based on their extreme preeclampsia presentation. The average age of the patients was 29.08 years, with a standard deviation of 5.81 years (range: 18-40). The average gestational age was 33.62 weeks, with a standard deviation of 2.18 weeks (range; 28-36). The mean gravidity was 3.80 , with a standard deviation of 1.93 . (range; 1 - 7). The average parity was 3.50 , with a standard deviation of 2.23. (range; 0 - 7) Table 1.

\begin{tabular}{|l|c|c|c|c|c|}
\hline & N & Minimum & Maximum & Mean & $\begin{array}{c}\text { Std. } \\
\text { Deviation }\end{array}$ \\
\hline Age & 50 & 18.00 & 40.00 & 29.08 & 5.81 \\
\hline Gravidity & 50 & 1.00 & 7.00 & 3.80 & 1.93 \\
\hline Parity & 50 & 0.00 & 7.00 & 3.50 & 2.23 \\
\hline $\begin{array}{l}\text { Gestation } \\
\text { al age }\end{array}$ & 50 & 28.00 & 36.00 & 33.62 & 2.18 \\
\hline
\end{tabular}

Table 1: Demographic characters of the observed women

\begin{tabular}{|l|c|c|c|}
\hline & Before & After & P value \\
\hline Systolic blood pressure & $174.02 \pm 8.48$ & $152.98 \pm 7.17$ & $0.001^{* *}$ \\
Diastolic blood pressure & $113.38 \pm 5.10$ & $99.98 \pm 6.11$ & $0.001^{* *}$ \\
Maternal heart rate & $78.72 \pm 6.22$ & $90.46 \pm 6.07$ & $0.001^{* *}$ \\
\hline
\end{tabular}

Data are expressed as mean \pm standard deviation. ${ }^{* *} \mathrm{p}<0.01=$ highly significant.

Table 2:Comparison of the mean value of the mother'sblood pressure and heart rate pre and post MgSO4 treatment.

Table (2) shows that a substantial change in pre and post MgSo4 administration, maternal blood pressure and heart rate were measured. 
Table(3) shows that a substantial change in umbilical artery Doppler parameters pre and post MgSo4 administration.

\begin{tabular}{l|c|c|c|}
\hline & Before & After & P value \\
\hline RI & $0.69 \pm 0.027$ & $0.60 \pm 0.031$ & $0.001^{* *}$ \\
PI & $1.11 \pm 0.071$ & $0.90 \pm 0.17$ & $0.001^{* *}$ \\
S/D & $3.39 \pm 0.22$ & $2.73 \pm 0.16$ & $0.001^{* *}$ \\
\hline
\end{tabular}

Table 3: Comparison between the mean values of umbilical artery Doppler parameters measured pre and post administration of MgSO4.

\begin{tabular}{|l|c|}
\hline Proteins in Urine(+) & Percent \% \\
\hline 2 & $26.7 \%$ \\
\hline 3 & $53.3 \%$ \\
\hline 4 & $20.0 \%$ \\
\hline Total & $100.0 \%$ \\
\hline
\end{tabular}

Table 4: Distribution of proteinuria measured by dipsticks in the studied group

\begin{tabular}{|l|c|c|}
\hline Baby born weight (gram) & N & $\mathbf{~ \% ~}$ \\
\hline Mean & 1990,52 & - \\
$<1500$ & 7 & 14 \\
$1500-1999$ & 9 & 18 \\
$2000-2499$ & 21 & 42 \\
$2500-2999$ & 8 & 16 \\
$>3000$ & 5 & 10 \\
\hline Apgar score & & \\
$0-3$ & 11 & 22 \\
$4-6$ & 26 & 52 \\
$7-10$ & 13 & 26 \\
\hline Fetal complication & & - \\
IUGR & 9 & - \\
IUFD & 5 & \\
\hline
\end{tabular}

Table (5): Neonatal Outcomes of patients with severe preeclampsia/eclampsia

\section{DISCUSSION}

Preeclampsia is a significant cause of maternal mortality globally, also a major eason of perinatal mortality and morbidity, affecting $2-8 \%$ of pregnant women ${ }^{8}$. It's characterized by high blood pressure and proteinuria, which appear after 20th week of pregnancy ${ }^{9}$.Preeclampsia can lead to eclampsia if it presents as hemolysis,high levelsof liver enzymes and a low platelet count (HELLP) syndrome.Both eclampsia and HELLP syndrome have been linked to serious side effects including: cerebral hemorrhage, lung edema, renal failure, and liver hemorrhage ${ }^{10}$.

Preeclampsia has been linked to an impaired trophoblastic invasion of maternal spiral arteries, leading to increased reliance on uterine artery traffic, according to extensive studies conducted in the last 15 years ${ }^{11}$.

Magnesium inhibits trans-membrane calcium transport, lowering calcium intake and reducing the contractile effects of vasoactive agents in vascular smooth muscle in the extracellular space. It also functions as a calcium antagonist inside the cell, modulating the vasoconstrictor effect ${ }^{12}$.

The current research looked at the fetal umbilical artery's Doppler velocimetry parameters (resistance index [RI], pulsatility index [PI], and systolic/diastolic [S/D] ratio) before and after a loading dose of magnesium sulfate was given to pregnant patients with extreme preeclampsia.

The study group's age ranged from 18 to 40 years, as shown in table (1), with a mean age of 29.08 years and a standard deviation of 5.81 .

Table 1 shows that the gestational age of the study group ranged from 28 to 36 weeks, with a mean gestational age of 33.62 weeks and a standard deviation of 2.18 weeks. In terms of the study group's gravidity, as shown in table (1). The standard deviation was 1.93 and the mean was 3.80.

In patients with extreme preeclampsia, 20 minutes of intravenous infusion of $6 \mathrm{gm}$ magnesium sulfate had a major hemodynamic effect, according to our findings. As seen in the tables(2)., pregnant women with extreme preeclampsia experienced a substantial decrease in systolic and diastolic blood pressure as well as a rise in heart rate after receiving a magnesium infusion.

These findings are consistent with those of Souza et al. 13, who found that acute magnesium sulfate administration lowers blood pressure and systemic vascular resistance while increasing cardiac output in women with preeclampsia.

Table (4) indicate that Proteinuria was classified into three levels: 26.7 percent had $(2+)$ proteinuria, 53.3 percent had $(3+)$ proteinuria, and $20 \%$ had $(4+)$ proteinuria.

As shown in table 3,Magnesium sulfate was given to pregnant women who had severe preeclampsia and eclampsia,Doppler velocimetry indices of the umbilical artery showed the significant decrease.

These findings are consistent with those of Souza et al. ${ }^{13}$, who found that intravenous magnesium sulfate treatment reduced the umbilical artery Doppler velocimetry indices (RI, PI, S/D) in pregnant women with preeclampsia.

Similarly, Houlihan et al. ${ }^{14}$ demonstrated that magnesium sulfate reduces umbilical artery vascular resistance, resulting in vasodilator effect.

Nonetheless, there was no substantial decrease in the vascular resistance of the umbilical artery, as assessed by the pulsatility index, in the Belfort et al. 15 study evaluating pregnant women with preeclampsia.

The point in the Belfort et al. ${ }^{15}$ study, that women were not stratified according to severity, and the majority of cases were moderate preeclampsia, may explain these conflicting findings.

Infusion of magnesium sulfate reduces the fetus RIumbilical after infusion of MgSO4, indicating that fetal blood flow has increased according to Nazanin et al., ${ }^{16}$

Doppler ultrasound examination has been shown to be effective in determining the fetal vascular status and a fetal blood supply in a number of studies ${ }^{17}$.

According to the findings of this and other studies, more systematic studies with larger populations, as well as a meta-analysisof all current studies' findingsare needed to support finding sconcerning MgSO4's effects on fetal blood flow on fetal blood flow $^{17}$. 
Kovac et al., ${ }^{18}$ concluded that magnesium sulfate reduced pulsatility index of the umbilical arteryby 0.01 , when given to women who were using it to avoid preterm labor and had normal blood pressure levels.

Belfort et al., ${ }^{15}$ found thatsystolic and diastolic pressures were decreased in pregnant women with hypertensive disorders by 14 and $11 \mathrm{mmHg}$ respectively, using magnesium sulfate, while maternal heart rate increased by 4 beats per minute, however there is no statistical significance The PI of umbilical arteries decreased by 0.02 in the same sample, but this was not statistically important.

Rantonen et al., ${ }^{19}$ showed that only women with extreme preeclampsia reported decreased systolic and diastolic blood pressure after receiving magnesium sulfate for convulsion prevention, while the maternal heart rate increased (p0.001). The authors discovered large reduction in PI of the umbilical $(p=0.008)$ with a reduction of 0.05 after using magnesium sulfate. It's worth noting that various reports on this subject are conflicting.

There was an important difference in the umbilical artery Doppler parameters of the study group before and after MgSO4 administration (mean values of RI, $\mathrm{PI}$, and S/D ratio in the study group), as shown in tables (3).

\section{CONCLUSION}

To diagnose abnormalities and evaluate fetal wellbeing in pregnant women with preeclampsia, clinical examinations should be backed up and verified by laboratory tests and ultrasonography. Doppler flow tests, which may help predict preeclampsia and determine fetal health, should be included in the sonographic examination.It has a vasodilator impact on the blood vessels of the mother and fetus.Several hemodynamic modifications have been observed after using magnesium sulfate. In patients with extreme preeclampsia, magnesium sulfate causes major changes in the umbilical artery Doppler indices. In patients with serious preeclampsia, magnesium sulfate should be provided.

\section{REFERENCES}

1. Cavalli RD, Korkes HA. Preeclampsia: New Concepts and Approach of a National Protocol. RevistaBrasileira de Ginecologia e Obstetrícia 2019; 41(5):277-9.

2. Phoswa WN, Naicker T, Ramsuran V. Preeclampsia: the role of highly active antiretroviral therapy and immune markers. Inflammation Research. 2019; 68(1):47-57.

3. ACOG committee on obstetric practice. ACOG practice bulletin. Diagnosis and management of preeclampsia and eclampsia. Number 202, January 2019. American college of obstetricians and gynecologists.Int J Gynaecolobstet .e 4-3, 2019.

4. Mathers CD. History of global burden of disease assessment at the World Health Organization.Archives of Public Health. 2020; 78(1):1-3.
5. Mira IM, Sedek AE, Abd EL-Aziz AF. Bleeding time after magnesium sulphate infusion in cases of preeclampsia.The Egyptian Journal of Hospital Medicine. 2019; 76(4):3957-62.

6. Abdel Aziz FA, Rady MS, Abd-Eltawab AM. Value of Middle Cerebral/Umbilical Artery Resistance Index Ratio in Neonatal Outcome in Patients with Intrauterine Growth Restriction (A Prospective Study).International Journal of Medical Arts. 2020; 2(4):828-34.

7. Maged AM, Hashem AM, Gad Allah SH. The effect of loading dose of magnesium sulfate on uterine, umbilical, and fetal middle cerebral arteries Doppler in women with severe preeclampsia: a case control study. Hypertension in Pregnancy. 2016; 35(1):91-9.

8. Steegers EA, von Dadelszen P, Duvekot JJ. Preeclampsia. Lancet. 2010; 3: 631-44.

9. MirzaieF, Maiz N, Bonino S. Pathology of preeclampsia. 2009; 135:487.

10. Van wijk M, Boer K, Van JA. Micro particle subpopAtion is increased in PE: possible involvement in vascular dysfunction. $A m \quad J$ ObstetGynecol. 2002; 187: 450.

11. Papageorghious AT, Yuck H. Second trimesteruterineartey Doppler screening in unselected population: a reviewJMatern-fetal Neonatal Med. 2002; 78-88.

12. Spencer AS, Noronha Neto C, Amorim MM. Magnesium sulfate s'ndromes in hypertensive pregnancy: maternal and fetal hemodynamic effects. Femina. 2006; 34(9): 625-31.

13. Souza AS, Amorim MM, Coutinho IC. Effect of the loading dose of magnesium sulfate (MgSO4) on the parameters of Doppler flow velocity in the uterine, umbilical and middle cerebral arteries in severe preeclampsia. Hypertension Pregnancy. 2010; 29(2): 123-34.

14. Houlihan DD, Dennedy MC, Ravikumar N. Antihypertensive therapy and the feto-placental circulation: effects on umbilical artery resistance. $J$ Perinat Med. 2004; 32:315-9.

15. Belfort MA, Saade GR, Grunewald C. Change in estimated cerebral perfusion pressure after treatment with nimodipine or magnesium sulfate in patients with preeclampsia. Am J ObstetGynecol .1999; 181: 4027

16. Nazanin F, Negin R, Saeed M. Effect of Magnesium Sulfate on Doppler Parameters of Fetal Umbilical and Middle Cerebral Arteries in Women with Severe Preeclampsia. J Clin Imaging Sci. 2012; 2: 85.

17. Nanthakomon T, Uerpairojkit B. Outcome of smallfor-gestational-age fetusesaccording to umbilical artery Doppler: is there any yield from additional middle cerebral artery Doppler. J Matern Fetal Neonatal Med. 2010; 23(8):900-5.

18. Kovac CM, Howard BC, Pierce BT. Fetoplacental vascular tone is modified by magnesium sulfate in the preeclamptic ex vivo human placental cotyledon. Am J ObstetGynecol. 2003; 189 (3):839-42.

19. Rantonen T, Kääpä P, Grönlund J. Maternal magnesium sulfate treatment is Associated with Reduced brain-blood flow perfusion in preterm infants. Crit Care Med. 2001; 29 (7):1460-5. 